\title{
Nossos erros me afetam? Efeito de informações negativas na identificação com endogrupo e na autoestima'
}

\author{
Raquel Loewenhaupt* \\ Ronaldo Pilati
}

Universidade de Brasília, Instituto de Psicologia. Brasília, DF, Brasil

\begin{abstract}
Resumo: Teorias clássicas sobre consistência cognitiva recentemente têm sido exploradas enquanto processos não apenas intraindividuais, mas influenciados por aspectos grupais. Tendo em vista a tendência humana ao enaltecimento pessoal e grupal e a teoria unificada de cognição social implícita, informações negativas a respeito do endogrupo têm potencial de gerar inconsistência por meio da dissonância cognitiva e de desbalanceamento. Para testar as funções protetivas da consistência cognitiva diante das ameaças à avaliação positiva do endogrupo, 156 participantes passaram por pré e pós-teste de autoestima e por manipulação experimental de ameaça ou neutra. Não houve diferença significativa na autoestima dos participantes, nem no estado afetivo. Nos grupos experimentais, embora apontassem os comportamentos como negativos, muitos alegaram ser esperteza e jeitinho brasileiro. Pesquisas sobre dissonância apontam que, quando o objeto da redação contra-atitudinal é uma norma cultural, os efeitos da dissonância e a possibilidade de mudança de atitude são minimizados. Resultados serão discutidos.
\end{abstract}

Palavras-chave: consistência cognitiva, dissonância cognitiva, autoestima implícita.

Atitude é a associação de um objeto ou grupo social a um atributo/valência. Já a autoestima é uma atitude que refere-se à associação do conceito de si a um atributo/valência (Greenwald, Rudman, Farnham, Nosek, \& Mellott, 2002). Por sua vez, o autoconceito é a associação entre o conceito de si e outros conceitos (Schnabel \& Asendorpf, 2010). A atitude em relação ao endogrupo, a autoestima e o autoconceito são associados com implicações em processos de manutenção da consistência cognitiva e a compreensão dessas associações implica em maior entendimento sobre fenômenos sociais (Greenwald et al., 2002).

A teoria da identidade social (Tajfel, 1978) aponta que o pertencimento ao grupo é a dimensão mais relevante da visão de si. As quatro categorias básicas de grupos são: grupos primários (como família e amigos próximos), grupos sociais (como trabalhadores de uma empresa, grupos de estudo), associações (vizinhos em bairros grandes, estudantes em instituições grandes) e categorias (pessoas de mesma nacionalidade e/ou do mesmo sexo) (Forsyth \& Burnette, 2010). Os diferentes tipos de grupos apresentam diferenças quanto à entitatividade - fatores perceptuais como similaridade, proximidade e destino comum que influenciam tanto a percepção de membros quanto de não membros em relação à unidade do grupo (Campbell, 1958).

As pessoas tendem a ter atitude positiva avaliar positivamente - os grupos dos quais fazem

\footnotetext{
Endereço para correspondência: raquelrss.unb@gmail.com

1 Pesquisa conduzida com apoio de bolsa de mestrado da Capes vinculada a Raquel Loewenhaupt e de bolsa produtividade do CNPq vinculada a Ronaldo Pilati.
}

parte (Tajfel, 1978) e, portanto, informações negativas sobre o endogrupo são inconsistentes com a manutenção dessa atitude positiva. O ser humano, no entanto, não lida bem com inconsistências. Teorias clássicas sobre consistência cognitiva, como a teoria da dissonância cognitiva (Festinger, 1957) e a teoria do balanceamento (Heider, 1958), possuem como pressuposto fundamental que inconsistências entre cognições são desagradáveis, instáveis e tendem ao equilíbrio (Fiske \& Taylor, 2013).

A tendência humana de manter a consistência cognitiva é tida como princípio básico do pensamento humano e exerce papel fundamental na forma como as informações sociais são processadas (Gawronski \& Strack, 2012). Essa tendência é atualmente compreendida como sendo associada a processos cognitivos e motivacionais (Baumeister, 2010) e se relaciona a processos de defesa do self(Greenwald \& Ronis, 1978). O objetivo deste trabalho é testar o efeito de informações negativas sobre o endogrupo na identificação com o grupo e na autoestima.

\section{Teorias sobre consistência cognitiva e influência de aspectos sociais na manutenção da consistência}

A teoria da dissonância cognitiva indica que entrar em contato com informações inconsistentes com atitudes prévias cria uma ameaça para o senso de si, que precisa ser reduzida (Gawronski \& Strack, 2012). A dissonância cognitiva é negativa, desconfortável e fisiologicamente excitatória (Cooper, 2007). Sua resolução pode ocorrer com a mudança de atitude (Fiske \& Taylor, 2013), mas, quando mudar de atitude, pode ser uma ameaça à visão 
de si, outros processos de resolução podem ocorrer como: 1) a distorção de informações que sejam conflitantes com a manutenção de uma auto avaliação positiva (Cooper, 2007); e 2) o engajamento em estratégias de enaltecimento pessoal e do endogrupo quando em situações de comparação social (Vignoles, Regalia, Manzi, Golledge, \& Scabini, 2006).

Dissonância cognitiva da forma concebida por Festinger (1957) possui componente social substancial que não recebeu atenção até recentemente, uma vez que a maioria dos estudos a conceitualiza como processo intraindividual (McKimmie, 2015). Clémence (1994) aponta que há dois níveis de conflito eliciado pelo comportamento contraatitudinal - procedimento experimental mais utilizado para criar a inconsistência que provoca a dissonância cognitiva (Cooper, 2007): nível intraindividual e nível intergrupal. Este último cria motivação para proteger a identidade social e limita a habilidade de redução da dissonância pela mudança de atitude. Levando em consideração a associação da identidade pessoal e social na constituição do autoconceito como central nos processos de dissonância, Glasford, Dovidio e Pratto (2008) fizeram dois experimentos e encontraram resultados que sugerem que pessoas que possuíam uma crença pessoal importante violada pelo comportamento do grupo reduziam a dissonância diminuindo a identificação com o grupo ou engajando em ativismo em favor dessa crença. Assim, a possibilidade de diminuição da identificação com o grupo existe quando uma crença pessoal muito importante é violada pelo grupo. Quando esse não era o caso, a resolução da inconsistência se deu por meio de estratégias de enaltecimento da identidade social, quão maior fosse a identificação com o grupo.

Outra teoria sobre consistência cognitiva é a teoria do balanceamento de Heider (1958) que, assim como a teoria da dissonância cognitiva, postula sobre a seletividade da apreensão de informações com objetivo de autoproteção e manutenção da consistência cognitiva (Fiske \& Taylor, 2013). Ela diverge da teoria da dissonância cognitiva, pois aponta a formação de estruturas cognitivas de balanceamento que associam tríades de conceitos e atributos, tendo implicações não apenas no armazenamento, mas na recuperação de memórias (Fiske \& Taylor, 2013).

A compreensão sobre automaticidade introduzida por Bargh (1994) de que as pessoas se engajam, sem escolher conscientemente, tanto em processos controlados quanto em automáticos (Calrston, 2010) e de que processos automáticos influenciam motivações que desencadeiam cognições e comportamentos (Fiske \& Taylor, 2013) implicou em desdobramentos metodológicos, com a criação de instrumentos indiretos de mensuração de atitude, e teóricos, com novos desdobramentos a respeito da consistência cognitiva (Petty, Briñol, \& Johnson, 2012).

A teoria unificada de cognição social implícita proposta por Greenwald et al. (2002) integra construtos cognitivos e afetivos a partir de três influências: 0 crescente interesse em automaticidade e cognição implícita, o desenvolvimento do Teste de Associação Implícita (Greenwald, McGhee, \& Schwarts, 1998), e as teorias de consistência cognitiva dos anos 1950, em especial a teoria do balanceamento de Heider (1958). Essa teoria usa três termos comuns em teorias modernas sobre network neural (Greenwald et al., 2002): conceitos (representando pessoas, grupos e atributos - valência positiva e negativa), força da associação (potencial de um conceito de ativar outro) e ativação de conceitos (excitação decorrente da associação existente entre um conceito com outro já ativo). Foi demonstrada, pela teoria, associação positiva de balanceamento entre autoestima, atitude em relação ao endogrupo e identificação com o grupo (Greenwald et al., 2002). Assim, quanto maior a autoestima e a identificação com o grupo, por consequência, maior a avaliação positiva em relação ao grupo, sendo, portanto, uma teoria que assume a relação próxima da autoestima e da identificação com o endogrupo para a manutenção da consistência cognitiva. Este trabalho testa o efeito de ameaça à manutenção da avaliação positiva em relação ao endogrupo nos demais elementos da estrutura cognitiva de balanceamento que os envolve, autoestima e identificação com o grupo, uma vez que é esperado a ocorrência de inconsistência de acordo com os pressupostos da teoria do balanceamento cognitivo (Heider, 1958). As categorias sociais utilizadas nas pesquisas da teoria unificada de cognição social implícita (Greenwald et al., 2002), homens e mulheres fazem parte do mesmo tipo básico de um dos grupos deste trabalho - brasileiros - e são diferentes do outro grupo - estudantes universitários (Forsyth \& Burnette, 2010). O critério da escolha foi estes serem grupos dos quais todos os participantes-alvo da pesquisa são membros. Não há restrições de tipos de grupos que se enquadram ou não na efetividade e existência da estrutura de balanceamento envolvendo atitude em relação ao endogrupo, identificação com o grupo e autoestima. A estrutura de balanceamento identificada na teoria unificada de cognição social implícita é potencialmente aplicável a qualquer grupo (Greenwald et al., 2002).

\section{Especificidades da autoestima na consistência cognitiva}

A autoestima é associada à identificação com grupos (Stets \& Burke, 2013) eé parte da estrutura de balanceamento investigada nesse trabalho (Greenwald et al., 2002). Sua conceituação também foi impactada com o advento do conhecimento a respeito de automaticidade, uma vez que as pessoas processam as informações sociais não apenas explicitamente, mas também de forma implícita (Bargh, 1994), e o self atua de forma diferente nesses dois modos de processamento (Greenwald \& Farnham, 2000). Hoje, conceituam-se duas categorias para autoestima: a explícita, que se refere a avaliações conscientes e deliberadamente fundamentadas; e autoestima implícita, que se refere a avaliações altamente eficientes que ocorrem de forma não 
intencional e fora da atenção consciente (Jordan, Spencer, Zanna, Hoshino-Browne, \& Correll, 2003).

Uma das prerrogativas menos contestadas em psicologia social é a de que as pessoas são motivadas a proteger e aumentar sua autoestima (Vignoles et al., 2006). Rudman, Dohn e Fairchild (2007) apontam que a autoestima participa em processos automáticos de defesa tendo em vista seu funcionamento contra ameaças ao autoconceito. Eles demonstraram, em quatro experimentos, que a compensação da autoestima implícita é um processo de defesa do self $\mathrm{em}$ resposta a ameaças, que ocorre espontaneamente e sem esforço, mantendo a consistência cognitiva e funcionando como um regulador de afeto.

Ademais a autoestima implícita estar relacionada ao processo de manutenção da consistência cognitiva, há ainda outra questão que influencia esse processo: a ambivalência implícita - quando avaliações implícitas e explícitas em relação a algo diferem (Petty et al., 2012). Enquanto o princípio de balanceamento parece operar em um nível primário do pensamento, a ambivalência implícita está relacionada a processos secundários, metacognitivos. Isso porque quando uma pessoa nega uma reação avaliativa ou considera que se refere a outra fonte (que não à sua visão pessoal) ela não a utiliza em auto-relatos deliberados, mas esta avaliação ainda pode afetar processos automáticos. Então, a medida explícita acessa apenas a associação endossada pela pessoa, enquanto a medida indireta pode identificar avaliações existentes, que a pessoa não endossa conscientemente (Petty et al., 2012).

Jordan et al. (2003) apontam para a existência de dois tipos de alta autoestima. Uma chamada de alta autoestima segura, bem fundamentada, e a outra, chamada de alta autoestima defensiva, que teria características frágeis e suscetíveis a ameaças. Esta última seria decorrente da manutenção simultânea de duas atitudes discrepantes em relação ao self: sentir-se bem, em um nível consciente e explícito, e relativamente mal, em um nível implícito. Os autores obtiveram resultados que indicam que as pessoas com alta autoestima defensiva tendem a engajar-se em estratégias de autoenaltecimento por meio de viés em relação ao endogrupo, e a apresentar mais justificações para suas respostas, que as pessoas que possuem alta autoestima segura (explícita e implícita, altas). A autoestima defensiva é ainda associada à maior tendência à distorção de informações nocivas ao self (Kernis, Lakey, \& Heppner, 2008) e maior desconforto diante de ameaças (Jordan et al., 2008). Sendo a alta autoestima defensiva um exemplo de ambivalência implícita (Petty et al., 2012), é importante levar isso em consideração em pesquisas que exploram processos de defesa mediante inconsistências, tendo em vista as características de defensividade apontadas.

$\mathrm{O}$ efeito de ameaça à consistência cognitiva na tríade de balanceamento da teoria unificada de cognição social implícita, considerando o potencial efeito moderador da ambivalência implícita não foi, da forma proposta, outrora testado na literatura. Este trabalho visa, também, contribuir metodologicamente com a elaboração do Teste de Associação Implícita para emprego em pesquisas intraculturais, além de fomentar a compreensão de processos grupais no Brasil, para além da incorporação de resultados oriundos de pesquisas realizadas em outros países.

Considerando as tendências humanas de avaliar positivamente os grupos dos quais faz parte (Tajfel, 1978), de proteção e elevação da autoestima (Vignoles et al., 2006) e de manutenção da consistência cognitiva como princípio básico do processamento de informações (Gawronski \& Strack, 2012) e, ainda, tendo em vista o caráter defensivo da busca por consistência cognitiva (Cooper, 2007), é objetivo deste trabalho: testar o efeito de informações negativas sobre o endogrupo na identificação com o grupo e na autoestima. As hipóteses são:

H1: Os participantes expostos a texto contendo ameaça à avaliação positiva do grupo distorcerão as informações, avaliando-as como menos negativas.

H2: Os participantes expostos a texto contendo ameaça à avaliação positiva do grupo apresentarão maiores escores de afetos negativos.

H2a: Entre esses participantes expostos ao texto, os com alta autoestima defensiva apresentarão escores maiores de afetos negativos.

H3: Os participantes expostos ao texto contendo ameaça à avaliação positiva do grupo apresentarão escores de autoestima implícita no pós-teste maiores que os do pré-teste (compensação da autoestima implícita).

H3a: Entre esses participantes expostos ao texto, aqueles com alta autoestima defensiva apresentarão maior compensação da autoestima implícita.

H4: Participantes em que a mensuração da identificação com o grupo tenha sido feita antes da exposição ao texto contendo ameaça à avaliação positiva do grupo terão escores maiores de identificação que os participantes em que a mensuração tenha sido feita depois.

\section{Método}

\section{Participantes}

Participaram do estudo 156 estudantes universitários selecionados por conveniência; 18 foram retirados por terem redigido resposta contrária às instruções. Dos 138 restantes, $75,4 \%$ eram mulheres. A idade dos participantes variou de 17 a $35 \operatorname{anos}(M=20,21, D P=2,55)$.

\section{Instrumentos}

Escala de autoestima de Rosenberg: a escala de Rosenberg (1965) foi utilizada para avaliar a autoestima global. Esse instrumento transformou-se, ao longo de mais de quatro décadas, numa referência, sendo a escala de avaliação da autoestima global mais utilizada na investigação psicológica (Zeigler-Hill \& Jordan, 2010). Dez 
itens classificados pelo participante em escala de resposta de quatro pontos ( $\alpha$ pré-teste $=0,86 ; \alpha$ pós-teste $=0,87$ ).

Teste de Associação Implícita - TAI (Greenwald \& Farnham, 2000): para mensuração da autoestima implícita $(\alpha$ pré-teste $=0,73 ; \alpha$ pós-teste $=0,67)$.

O TAI (Greenwald et al., 1998) é utilizado como instrumento para mensuração de atitudes implícitas e foi desenvolvido como um desdobramento metodológico decorrente da demanda por aferição de cognições automáticas (Calrston, 2010). O TAI mensura a força da associação entre conceitos e atributos, considerando o tempo de latência da resposta, e possui a premissa de que quanto mais fácil uma tarefa mental, mais rápida a tomada de decisão e menor a quantidade de erros. É um instrumento utilizado em muitas áreas como psicologia cognitiva, social, forense, clínica, neuropsicologia, pesquisas de mercado etc. (Lane, Banaji, Nosek, \& Greenwald, 2007). Um TAI de autoestima, por exemplo, indica que a pessoa possui autoestima implícita alta se as respostas forem mais rápidas e com menos erros quando as categorias autorreferentes e atributos positivos estiverem juntos do que quando as categorias autorreferentes e atributos negativos estiverem juntos.

Foram utilizados os estímulos para as categoriasalvo - eu e não eu - propostos por Modesto (2014) e Modesto e Pilati (2015). Os estímulos utilizados para os atributos, positivo e negativo, foram escolhidos a partir de lista de palavras classificadas em função de normas de valência para a língua portuguesa (Oliveira, Janczura \& Castilho, 2013). Após a confecção do TAI de autoestima, realizou-se uma testagem piloto com 113 participantes apenas para identificação da adequabilidade da medida construída para utilização $(\alpha=0,65)$. A idade dos participantes variou de 18 a $49 \operatorname{anos}(M=21,65, D P=5,158)$ e $70,8 \%$ eram mulheres. A correlação entre o bloco um e o bloco dois do instrumento foi $r=0,483, p<0,001$. Um viés que pode ser prejudicial ao TAI é a influência do primeiro bloco de testes sobre o segundo (Lane et al., 2007), por isso é mais adequado encontrar uma correlação moderada entre os dois blocos.

Escala de identificação com o grupo: tradução adaptada de Leach et al. (2008). Catorze itens classificados pelo participante em uma escala de sete pontos, que podem ser adaptados para utilização em investigação de identificação com qualquer grupo (ver Apêndice A). Nesta pesquisa, os grupos escolhidos foram brasileiros $(\alpha=0,91)$ e estudantes universitários $(\alpha=0,79)$. A escala possui cinco componentes (Leach et al., 2008): Solidariedade (itens 3, 8 e 14; indica a ligação psicológica e comprometimento sentido em relação a membros do grupo); Satisfação (itens 1, 2, 5 e 9; indica a manutenção de uma avaliação positiva em relação ao grupo); Centralidade (itens 6, 10 e 13; indica a saliência e importância atribuídas ao pertencimento ao grupo); Autoestereótipo (itens 7 e 11; indica o quanto a pessoa percebe a si como similar a um típico membro do grupo); e Homogeneidade (itens
4 e 12, indica o quanto a pessoa percebe o grupo como homogêneo e distinto de outros grupos).

Escala de estado afetivo: tradução adaptada da escala utilizada por Norton, Monin, Cooper e Hogg (2003) em pesquisa sobre dissonância cognitiva. Doze itens classificados pelos participantes em escala de sete pontos, contemplando afeto pessoal positivo $(\alpha=0,83)$, desconforto psicológico global $(\alpha=0,62)$ e afeto negativo autodirecionado $(\alpha=0,92)$. A instrução para resposta à escala era referente ao momento em que a escala estava sendo respondida, para checagem da dissonância cognitiva. Assim, a instrução era: "Responda aos itens seguintes indicando, em uma escala de 1 a 7 , em que $1=$ Nem um pouco e $7=$ Extremamente. Não há respostas certas ou erradas. Para cada item, escolha o valor na escala que melhor represente o que você está sentindo. Qualquer valor na escala pode ser utilizado. Utilize o cursor e clique sobre a opção escolhida". Os termos para a dimensão "afeto pessoal positivo" eram: energizado, amigável, bem, otimista e feliz. Para a dimensão "desconforto psicológico global", eram: inquieto, entediado e desconfortável. Para a dimensão "afeto negativo autodirecionado": nervoso comigo mesmo, irritado comigo mesmo, insatisfeito comigo mesmo e aborrecido comigo mesmo. A ordem dos termos seguiu a proposta por Norton et al. (2003).

\section{Procedimento}

Aos participantes, foi apresentado o Termo de Consentimento Livre e Esclarecido, com informações relativas à duração média da pesquisa, ao laboratório ao qual estava vinculada, ao objetivo, à informação sobre o sigilo da participação e destino dos dados obtidos, à indicação da necessidade de ler as instruções de cada etapa com atenção, ao caráter voluntário da participação e à possibilidade de desistência a qualquer momento. A pesquisa foi conduzida individualmente, no computador, por meio do software Inquisit versão 4.0.

$\mathrm{O}$ delineamento experimental consistia em três grupos: brasileiros, estudantes universitários e controle. A designação dos participantes aos grupos foi aleatorizada pelo software utilizado. Em todos os grupos, os participantes passavam por pré e pós-teste de mensuração de autoestima implícita e explícita, sendo que o pós-teste era a reaplicação das medidas. Em pesquisa sobre confiabilidade teste-reteste, o teste de Rosemberg $(r=0,80)$ e o TAI $(r=0,69)$ mostraram as melhores estabilidades ao longo do tempo dentre outros testes de autoestima (Bosson, Swann, \& Pennebaker, 2000).

Nos dois grupos, a manipulação experimental consistiu em os participantes lerem uma notícia fictícia e negativa sobre grupo do qual eles fazem parte, sendo convidados a elencar características do grupo que levaram à sua protagonização na notícia, além de escrever um parágrafo curto, direcionado a pessoas de outros grupos, alertando sobre as características 
negativas do grupo - comportamento contra-atitudinal (ver Apêndices B e C). Foram utilizados nomes de instituições nas notícias fictícias apenas para aumentar a percepção de veracidade, não tendo elas elaborado ou divulgado esses conteúdos. Depois, os participantes avaliaram a notícia (como negativa, neutra ou positiva - para aferição da valência atribuída à informação) e responderam ao instrumento para mensuração de estado afetivo (Panas). Além disso, responderam a uma escala de identificação com o grupo, com apresentação aleatorizada: antes ou depois da manipulação experimental. No grupo controle, os participantes leram uma notícia verídica sobre a descoberta de uma nova espécie de peixe (ver Apêndice D), avaliaram a notícia - como negativa, neutra ou positiva - e responderam ao instrumento de mensuração de estado afetivo. Todos os participantes, ao fim, responderam aos itens sociodemográficos. Foi perguntado aos participantes se julgaram os textos das notícias claros e convincentes. Por fim, realizaram-se o esclarecimento e o agradecimento pela participação.

Para analisar a associação entre autoestima implícita e explícita a amostra foi dividida em três grupos, equivalentes a aproximadamente $33 \%$ dos respondentes, cada. Os participantes foram categorizados como possuindo alta autoestima segura (autoestima explícita e implícita altas) quando seus escores nas duas medidas estavam no intervalo referente a pontuações mais altas; como possuindo alta autoestima defensiva (autoestima explícita alta e implícita baixa) quando seu escore da medida explícita estava no intervalo referente a pontuações mais altas e da medida indireta no intervalo referente a pontuações mais baixas; e como possuindo autoestima moderada congruente quando apresentavam os escores nas duas medidas de autoestima no intervalo referente a pontuações medianas.

A quantidade de participantes em cada categoria de autoestima, nos grupos experimentais, foi equilibrada (13 participantes com autoestima insegura, $12 \mathrm{com}$ segura e 12 com moderada congruente). Os demais participantes apresentaram, então, associações entre a autoestima implícita e explícita sem efeitos, apontados na literatura, como diferenciadores mediante resolução de inconsistências.

\section{Resultados}

Para cálculo do escore D - resultado do TAI - foram excluídos os trials com latência de resposta menor que $400 \mathrm{~ms}$ e maior que $10.000 \mathrm{~ms}$, conforme recomendações da área (Greenwald, Banaji \& Nosek, 2003). Para possibilitar comparações entre o TAI e a escala de Rosenberg, os escores das duas medidas foram padronizados para uma escala variando de 0 a 4 . As duas medidas apresentaram correlação baixa, positiva e não significativa no préteste $(r=0,104, p=0,225)$ e no pós-teste $(r=0,079$, $p=0,354)$.
H1: Os participantes expostos a texto contendo ameaça à avaliação positiva do grupo distorcerão as informações, avaliando-as como menos negativas

Foram atribuídos os valores 1, 2 e 3 para as avaliações dadas como negativas, neutras e positivas, respectivamente. Assim, ao analisar a média das avaliações por grupo, foi possível verificar a tendência geral dessas avaliações. A notícia foi mais positivamente avaliada pelos participantes do grupo controle $(M=2,39, D P=0,60)$ que pelos do grupo experimental brasileiros $(M=1,42$, $D P=0,65)$ e estudantes $(M=1,21, D P=0,47)$. Essa diferença foi significativa, $F(2,135)=55,179, p<0,001$, $w^{2}=0,44$. Assim, a avaliação feita pelos participantes do grupo controle ficou, em média, entre neutra e positiva, e a dos grupos experimentais, entre negativa e neutra.

Realizou-se teste post-hoc Bonferroni que identificou que a diferença na avaliação entre o grupo experimental brasileiros e estudantes não foi significativa $(p=0,30)$. Nesses grupos, 64 participantes avaliaram as notícias como negativa $(73,6 \%), 18$ como neutra $(20,7 \%)$ e 5 como positiva (5,7\%). Embora a maior parte dos participantes desses grupos tenham avaliado as notícias como negativas, menos participantes avaliaram a notícia como neutra ou positiva entre os que responderam à escala de identificação com o grupo antes da exposição ao texto contendo ameaça à avaliação positiva do endogrupo $(M=1,23, D P=0,48)$ que entre os que responderam à escala depois $(M=1,42, D P=0,66)$, mas essa diferença não foi significativa, $\mathrm{F}(1,86)=2,4, p=0,125$, $\eta^{2} p=0,027$.

Em análises considerando os componentes da escala de identificação, a forma como a notícia foi avaliada não teve implicações significativas em nenhum componente da identificação para os participantes que responderam à escala de identificação após a exposição aos textos contendo ameaça à avaliação positiva do grupo. Para os participantes que responderam à escala antes, $\mathrm{o}$ único componente que apresentou efeito significativo na avaliação da notícia foi a satisfação, $F(1,44)=3,123$, $p=0,005, \eta^{2} p=0,575$.

Não foi identificada distorção da valência das informações negativas (H1) enquanto processo de defesa para manutenção da consistência cognitiva (Cooper, 2007).

H2: Os participantes expostos a texto contendo ameaça à avaliação positiva do grupo apresentarão maiores escores de afetos negativos

$\mathrm{O}$ estado afetivo não foi significativamente diferente a depender da condição experimental, $V=0,07$, $F(3,133)=1,758, p=0,10, \eta^{2} p=0,038$. Esse resultado indica que a informação negativa não teve implicação significativa no estado afetivo como hipotetizado (H2). Como apresentado na Tabela 1, na dimensão afeto pessoal positivo, a maior média foi encontrada no grupo brasileiros, seguido pelo grupo controle, e estudantes. $\mathrm{Na}$ dimensão desconforto psicológico global, a maior média foi encontrada no grupo estudantes, seguido pelo 
grupo brasileiros, e controle. Na dimensão afeto negativo autodirecionado, a maior média foi encontrada no grupo controle, seguida pelo grupo brasileiros, e estudantes.

Em análises considerando os componentes da escala de identificação, para os participantes que responderam à escala de identificação antes, a centralidade teve efeito significativo no afeto pessoal positivo, $F(1,44)=2,28, p=0,03, \eta^{2} p=0,55$. A correlação entre centralidade e afeto pessoal positivo foi positiva, baixa e não significativa, $r=0,228, p=0,14$, indicando tendência dos participantes que apresentaram escores maiores de centralidade antes apresentarem escores maiores de afeto pessoal positivo depois da exposição aos textos contendo ameaça à avaliação positiva do grupo.

Para os participantes expostos ao texto contendo ameaça à avaliação positiva do grupo antes de responder à escala de identificação, a dimensão desconforto psicológico global apresentou efeito significativo na centralidade, $F(1,42)=4,587, p<0,001, \eta^{2} p=0,696$. A correlação entre desconforto psicológico global e centralidade foi positiva, moderada e significativa, $r=0,457$, $p=0,002$, indicando tendência dos participantes que indicaram escores maiores de desconforto psicológico global apresentarem, em seguida, escores maiores de centralidade.

H2a: Entre os participantes expostos ao texto negativo, os com alta autoestima defensiva apresentarão escores maiores de afetos negativos

Nos grupos experimentais o tipo de autoestima não apresentou diferença significativa no estado afetivo, $V=0,28, F(6,66)=1,80, p=0,11, \eta^{2} p=0,141$. A hipótese de que indivíduos com alta autoestima defensiva apresentariam maior desconforto diante de ameaças (Jordan et al., 2008) não foi corroborada (H2a). Conforme a Tabela 2, na dimensão afeto pessoal positivo, a maior média foi dos participantes com alta autoestima segura, seguidos pelos participantes com alta autoestima defensiva, e participantes com autoestima moderada congruente. $\mathrm{Na}$ dimensão desconforto psicológico global, a maior média foi encontrada nos participantes com alta autoestima defensiva, seguidos pelos participantes com autoestima moderada congruente, e participantes com alta autoestima segura. $\mathrm{Na}$ dimensão afeto negativo autodirecionado, a maior média foi encontrada nos participantes com autoestima moderada congruente, seguidos pelos participantes com alta autoestima segura, e participantes com alta autoestima defensiva.

H3: Os participantes expostos ao texto contendo ameaça à avaliação positiva do grupo apresentarão escores de autoestima implícita no pós-teste maiores que os do pré-teste (compensação da autoestima implícita)

Diferenciou-se a autoestima implícita subtraindo o escore do pós-teste pelo do pré-teste. Assim, quanto maior o valor da diferença, maior o aumento da autoestima implícita no pós teste e, quanto menor o valor, maior a diminuição no pós-teste. Não foi encontrado efeito significativo na diferença da autoestima implícita no pré e pós-teste a depender da condição experimental, $\mathrm{F}(2,135)=0,147, p=0,863, \eta^{2} p=0,002$. Os participantes, em média, apresentaram essa diferença negativa $(M=-0,166, D P=0,31)$ e em todos os grupos as diferenças foram negativas, indicando uma diminuição na autoestima implícita no pós-teste, independente da condição. Em análises considerando os componentes da escala de identificação, nenhum componente apresentou efeito significativo na diferença da autoestima implícita. Assim, não foi possível identificar o processo de defesa espontâneo diante de ameaças (Rudman et al., 2007), compensação da autoestima implícita, como hipotetizado (H3).

H3a: Dentre os participantes expostos ao texto negativo, participantes com alta autoestima defensiva apresentarão maior compensação da autoestima implícita

Nos grupos experimentais, a autoestima explícita do pré-teste $(M=2,87, D P=0,67)$ e do pós-teste $(M=2,86, D P=0,69)$ apresentaram correlação positiva, forte e significativa, $r=0,935, p<0,001$, e não apresentaram diferença significativa, $t(86)=0,522$, $p>0,05, r=0,056$. Já a autoestima implícita do préteste $(M=2,59, D P=0,43)$ e do pós-teste $(M=2,43$, $D P=0,39)$, apresentaram correlação positiva, moderadamente forte e significativa, $r=0,708, p<0,001$, e apresentaram diferença significativa, $t(86)=4,686$, $p<0,001, r=0,45$. Observa-se que a autoestima implícita no pós-teste foi, em média, menor que no pré teste. Essa diferença não foi significativa a depender do tipo de autoestima, $F(2,47)=0,482, p=0,62, \eta^{2} p=0,02$. Esse resultado indica que a tendência de indivíduos com alta autoestima defensiva a engajar-se em estratégias de autoenaltecimento mediante ameaças por meio de viés em relação ao endogrupo (Jordan et al., 2008) não foi identificada, sendo, portanto mais uma hipótese não corroborada (H3a). A menor diferença foi para os participantes com autoestima moderada congruente, seguida pelos participantes com alta autoestima defensiva e, a maior diminuição na autoestima implícita no pós teste, foi dos participantes com alta autoestima segura, como apresentado na Tabela 3.

H4: Participantes em que a mensuração da identificação com o grupo tenha sido feita antes da exposição ao texto contendo ameaça à avaliação positiva do grupo terão escores maiores de identificação que os participantes em que a mensuração tenha sido feita depois

Não foi encontrada diferença significativa na identificação com o grupo para os participantes que responderam à escala antes $(M=64,95, D P=11,73)$ e depois $(M=66,67, D P=12,19)$ da manipulação experimental, $F(1,86)=0,45, p=0,504, \eta^{2} p=0,005$. A média de identificação com o endogrupo dos participantes do grupo estudantes $(M=67,29, D P=9,92)$ foi maior que a média de identificação dos participantes do grupo brasileiros $(M=64,42, D P=13,49)$, sendo essa 
diferença não significativa, $F(1,86)=1,257, p=0,265$, $\eta^{2} p=0,015$.

Em análises considerando os componentes da escala de identificação, o contrabalanceamento desta (responder à escala antes ou depois da manipulação experimental) não teve efeito significativo nos escores dos componentes. No entanto, houve diferença significativa quanto ao grupo (brasileiros ou estudantes) em três componentes da escala de identificação: solidariedade, satisfação e centralidade.
No componente solidariedade a média do grupo brasileiros $(M=15,91, D P=2,93)$ foi significativamente maior que a do grupo estudantes $(M=13,43, D P=3,34)$, $F(1,86)=13,647, p<0,001, \eta^{2} p=0,138$. No componente satisfação, a média do grupo estudantes $(M=22,79$, $D P=3,8)$ foi significativamente maior que a do grupo brasileiros $(M=19,89, D P=4,2), F(1,86)=11,331$, $p=0,001, \eta^{2} p=0,118$. No componente centralidade, a média do grupo estudantes $(M=16,88, D P=2,94)$ foi significativamente maior que a do grupo brasileiros

Tabela 1. Média de estado afetivo, nas três dimensões, a depender do grupo.

\begin{tabular}{cccc}
\hline & Brasileiros & Estudantes & Controle \\
\hline Dimensão estado afetivo & $M(\mathrm{DP}) / 95 \% \mathrm{IC}$ & $M(\mathrm{DP}) / 95 \% \mathrm{IC}$ & $M(\mathrm{DP}) / 95 \% \mathrm{IC}$ \\
Afeto pessoal positivo & $23,67(6,32) /[22 ; 25,33]$ & $23,02(4,60) /[21,3 ; 24,75]$ & $23,33(5,79) /[21,77 ; 24,9]$ \\
Desconforto psicológico global & $10,24(3,72) /[9,13 ; 11,36]$ & $11(3,51) /[9,84 ; 12,16]$ & $9,76(4,05) /[8,71 ; 10,81]$ \\
Afeto negativo autodirecionado & $12,38(5,94) /[10,52 ; 14,24]$ & $12,02(6,55) /[10,1 ; 12,95]$ & $13,35(6,41) /[11,6 ; 15,1]$ \\
\hline
\end{tabular}

Nota: IC $=$ intervalo de confiança

Tabela 2. Média de estado afetivo, nas três dimensões, a depender do tipo de autoestima, nos grupos experimentais.

\begin{tabular}{cccc}
\hline & Autoestima segura & Autoestima moderada & Autoestima defensiva \\
\hline Estado afetivo & $M(\mathrm{DP})$ & $M(\mathrm{DP})$ & $M(\mathrm{DP})$ \\
Afeto pessoal positivo & $26,25(6,24)$ & $22,67(5,49)$ & $24,38(6,03)$ \\
Desconforto psicológico global & $8,58(3,75)$ & $10,42(3,23)$ & $10,62(3,86)$ \\
Afeto negativo autodirecionado & $11(5,32)$ & $12,42(5,90)$ & $8,92(5,54)$ \\
\hline
\end{tabular}

Tabela 3. Diferença da autoestima implícita a depender do tipo de autoestima nos grupos experimentais.

\begin{tabular}{ccc}
\hline Tipo de autoestima & $M(D P)$ & $95 \%$ IC \\
\hline Autoestima moderada & $-0,143(0,25)$ & {$[-0,306 ; 0,019]$} \\
Autoestima defensiva & $-0,144(0,26)$ & {$[-0,305 ; 0,016]$} \\
Autoestima segura & $-0,24(0,32)$ & {$[-0,446 ;-0,036]$} \\
\hline
\end{tabular}

$(M=13,20, D P=4,28), F(1,86)=21,539, p<0,001$, $\eta^{2} p=0,202$.

\section{Discussão}

Este artigo teve por objetivo testar o efeito de ameaça à avaliação positiva do grupo na autoestima e na identificação com o grupo. Tendo em vista a motivação humana de proteger e aumentar sua autoestima (Vignoles et al., 2006), a tendência a avaliar positivamente grupos dos quais faz parte e sendo a identidade social parte fundamental do autoconceito (Tajfel, 1978), informações negativas sobre grupos dos quais se é membro possui potencial para instauração de inconsistência cognitiva. O caráter defensivo da busca por consistência cognitiva, para evitar sentimentos aversivos e manter a integridade pessoal (Cooper, 2007) indica que, neste caso, a tendência à consistência cognitiva seguiria por processos que não ameaçassem a autoavaliação e a relação com o grupo: a distorção de informações (Cooper, 2007) e o engajamento em estratégias de enaltecimento (Vignoles et al., 2006).

Ao contrário do esperado, nenhuma das hipóteses propostas foi corroborada. A distorção de informação negativa (H1), enquanto processo de defesa para manutenção da consistência cognitiva (Cooper, 2007), não foi identificada. No entanto, para os participantes que responderam à escala de identificação com o grupo antes, o componente satisfação teve efeito significativo em como a notícia foi avaliada. A satisfação é um componente da identificação com o grupo que pode levar à desconsideração de eventos negativos e à resistência a informações negativas sobre o endogrupo na intenção de manter a avaliação positiva em relação ao grupo (Leach et al., 2008).

A informação negativa não teve implicação significativa no estado afetivo (H2). No entanto, o componente centralidade da escala de identificação com o grupo mostrou efeitos que não haviam sido hipotetizados. Esses resultados indicam que, mesmo 
após o contato com informação negativa acerca do endogrupo, o afeto positivo é maior, quão maior tenha sido a declaração anterior acerca da importância do pertencimento ao grupo. E que quão maior o desconforto diante de uma informação negativa acerca do endogrupo, maior a atribuição de importância dada ao pertencimento ao grupo depois.

Também não foi possível identificar o processo de autoenaltecimento espontâneo diante de ameaças (Rudman et al., 2007) - compensação da autoestima implícita (H3). Os participantes com alta autoestima defensiva não apresentaram as características diferenciadas mediante ameaças (Jordan et al., 2008), mas maior desconforto e tendência a engajar-se mais em estratégia de autoenaltecimento, que indivíduos com alta autoestima segura (H2a e H3a).

Os participantes que responderam à escala de identificação com o grupo antes da manipulação experimental não apresentaram escores significativamente maiores de identificação em comparação aos que responderam depois (H4). Essa diferença era esperada com base em Glasford et al. (2008), que indicam que pessoas com uma crença pessoal importante violada pelo comportamento do grupo podem reduzir a dissonância diminuindo a identificação com o grupo, engajando em ativismo em favor dessa crença ou engajando em estratégias de enaltecimento da identidade social, quão maior a identificação com o grupo. Esse resultado, no entanto, não é suficiente para afirmar se as informações contidas na manipulação experimental, a respeito do comportamento do grupo, violavam ou não crenças individuais importantes dos participantes. Como indicado nos resultados de H2, o componente centralidade apresentou efeitos que não haviam sido hipotetizados. Assim, apesar de não ter sido possível identificar diferença significativa na identificação com o grupo de forma geral, a importância dada ao pertencimento ao grupo (centralidade) apresentou potenciais efeitos de regulação do humor diante de ameaças à avaliação positiva do grupo. Por fim, foram encontradas diferenças significativas em componentes da identificação a depender do grupo (brasileiros ou estudantes), mas não houve diferença significativa entre eles nas variáveis estudadas (humor, avaliação da notícia e diferença da autoestima).

Embora tenham, de forma geral, identificado as notícias dos grupos brasileiros e estudantes como negativas, processos relacionados à resolução da inconsistência entre informações negativas sobre o grupo e manutenção de avaliação positiva do endogrupo podem ter ocorrido em outro momento da vida dos participantes. Na questão em que os participantes eram indagados sobre características do grupo que se relacionavam à protagonização do grupo na notícia, no grupo brasileiros, $73,08 \%$ das respostas apontavam características negativas, como desonestidade, egoísmo e hipocrisia. No entanto, em $71,59 \%$ das respostas os participantes também apontaram características dúbias quanto à valência negativa como malandragem, esperteza, jeitinho brasileiro e aproveitador. Em estudo com 171 participantes Fischer, Ferreira, Milfont e Pilati (2014) identificaram que brasileiros com maior identificação com o país eram mais propensos a endossar cenários de corrupção, particularmente quando expostos a priming de malandro devido à ativação de símbolos nacionais positivos e conotações moralmente ambíguas. Os autores apontam para a necessidade de estudos intraculturais de investigação da variabilidade dos processos psicológicos associados a esse efeito.

Os resultados obtidos, contrários ao que é postulado pelas teorias sobre consistência cognitiva, indicam que é possível que variáveis intraculturais estejam influenciando esses processos. As normas sociais têm papel importante para a consistência do grupo e para a visão da dissonância enquanto fenômeno relacionado à identidade social (Robertson, 2006). Robertson e Reicher (1997) apontam que normas podem reduzir dissonância quando elas validam o comportamento contra-atitudinal ou quando sugerem que a inconsistência entre atitude e comportamento é normativa. Tendo isso em vista, é possível que a inconsistência entre atitude (avaliação positiva do grupo) e comportamento (engajamento em comportamentos negativos pelos membros do grupo, como indicado nos cenários propostos) seja uma norma cultural. Um problema é que as possibilidades de mudança de atitude, decorrente da resolução das inconsistências (Fiske \& Taylor, 2013), se são contrárias a uma norma do grupo, tendem a ser inibidas (McKimmie, 2015). Normas culturais tendem a ser resistentes à mudança fazendo-se necessário compreender ou decifrar a cultura no intuito de decodificar motivos encobertos que explicam comportamentos individuais e coletivos tidos como naturais (Silva, Zanelli, \& Tolfo, 2013).

Outra possibilidade explicativa desses resultados é a possível não adequabilidade dos instrumentos para aferição do efeito. A única medida indireta utilizada foi para mensuração da autoestima implícita, mas a teoria unificada de cognição social implícita proposta por Greenwald et al. (2002) se refere a processos automáticos. Apesar de ainda existir pouca evidência acumulada a esse respeito, conhecimentos autorrelevantes e ameaças tendem a ser processadas automaticamente (Fiske \& Taylor, 2013) e há evidências de que, quando os sujeitos não têm habilidade de controlar suas respostas, como em respostas a medidas indiretas, a associação entre favoritismo em relação ao endogrupo e aumento da autoestima é mais prontamente observada (Smurda, 2006). A utilização de medidas indiretas pode evidenciar efeitos não captados pelos instrumentos utilizados nesta pesquisa, já que minimizaria as implicações do gerenciamento de impressão e do auto engano (Zeigler-Hill \& Jordan, 2010). São necessárias pesquisas futuras que utilizem instrumentos mais adequados para aferição do efeito, que explorem os componentes da identificação com o grupo na regulação de afeto e na avaliação de informações sobre o grupo de forma não categórica, além de outras possíveis variáveis culturais intervenientes sobre a relação de comportamentos negativos do endogrupo e seus efeitos na cognição humana. 


\section{Do our mistakes affect me? Effect of negative information in ingroup identification and self-esteem}

Abstract: Classical theories on cognitive consistency have recently been explored not only as intraindividual processes, but influenced by group aspects. Given the human tendency to personal and group enhancement and the unified theory of implicit social cognition, negative information about the ingroup has the potential of generating inconsistency through cognitive dissonance and imbalance. To test the protective functions of cognitive consistency considering the threats to the positive evaluation of the ingroup, 156 participants underwent pre- and post-test of self-esteem and by neutral or threat experimental manipulation. There was no significant difference in participants' self-esteem nor in their affective state. In experimental groups, although behaviors were pointed as negative, many claimed to be cunning and the jeitinho brasileiro (the Brazilian way of doing things). Research on dissonance point that, when the object of counterattidudinal writing is a cultural norm, the effects of dissonance and the possibility of change in attitude are minimized. Results will be discussed.

Keywords: cognitive consistency, cognitive dissonance, implicit self-esteem.

\section{Nos erreurs me touchent? Effet des informations négatives sur l'identification avec endogroupe et l'estime de soi}

Résumé: Les théories classiques de la cohérence cognitive ont été récemment exploré les processus non seulement intraindividuelle, mais influencé par les aspects de groupe. Compte tenu de la tendance humaine à agrandissement personnel et de groupe et théorie unifiée de la cognition sociale implicite, des informations négatives sur l'endogroupe a le potentiel pour générer incohérence en dissonance cognitive et de déséquilibre. Pour tester les fonctions de protection de la cohérence cognitive contre les menaces à l'évaluation positive de l'endogroupe, 156 participants ont subi l'estime de soi pré- et post-test et manipulation expérimentale de la menace ou neutre. II n'y avait pas de différence significative dans l'estime de soi des participants, ou l'état affectif. Dans les groupes expérimentaux, bien que les comportements pointus comme négatif, beaucoup prétendu être moyen ruse et jeitinho brasileiro. La recherche sur la dissonance souligner que lorsque l'attitude contre l'écriture de l'objet est une norme culturelle, les effets de la dissonance et la possibilité d'un changement d'attitude sont minimisés. On discute les résultats.

Mots-clés: la cohérence cognitive, la dissonance cognitive, estime de soi implicite.

\section{Nuestros errores me afectan? Efecto de la información negativa sobre la identificación con el grupo y en la autoestima}

Resumen: Teorías clásicas sobre consistencia cognitiva recientemente han sido exploradas como procesos no solo intraindividuales, pero también influenciados por aspectos grupales. Reconociendo la tendencia humana al engrandecimiento personal y grupal y la teoría unificada de la cognición social implícita, informaciones negativas sobre el endogrupo tienen el potencial para generar inconsistencia por intermedio de la disonancia cognitiva y del desequilibrio. Para probar las funciones de protección de la consistencia cognitiva contra amenazas a la evaluación positiva del endogrupo, la autoestima de 156 participantes fue medida antes y después de la manipulación experimental de amenaza o neutra. No hubo diferencia significativa en la autoestima de los participantes, o en el estado afectivo. En los grupos experimentales, aunque consideraban los comportamientos como negativos, muchos dijeron que era astucia y el jeitinho brasileiro. Investigaciones sobre disonancia indican que cuando el objetivo de la redacción contra actitudinal es una norma cultural, los efectos de la disonancia y la posibilidad de cambio de actitud son minimizados. Resultados son discutidos.

Palabras clave: consistencia cognitiva, disonancia cognitiva, autoestima implícita.

\section{Referências}

Bargh, J. A. (1994). The four horsemen of automaticity: awareness, intention, efficiency and control in social cognition. In R. S. Wyer Jr. \& T. K. Srull(Eds.), Handbook of social cognition Basic Processes (pp. 153-208). Hillsdale, NJ: Lawrence Erlbaum Associates.

Baumeister, R. F. (2010). Social psychologists and thinking about people. In R. F. Baumeister \& E. J.
Finkel (Eds.), Advanced social psychology: the state of the science (pp. 5-24). Nova York, NY: Oxford University Press.

Bosson, J. K., Swann, W. B., Jr., \& Pennebaker, J. (2000). Stalking the perfect measure of implicit self-esteem: the blind men and the elephant revisited? Journal of Personality and Social Psychology, 79, 631-643. 
Campbell, D. T. (1958). Common fate, similarity, and other indices of the status of aggregates of persons as social entities. Behavioral Science, 3, 14-25.

Carlston, D. (2010). Social cognition. In R. F. Baumeister \& E. J. Finkel (Eds.), Advanced social psychology: the state of the science (pp. 63-99). Nova York, NY: Oxford University Press.

Clémence, A. (1994). Protecting one's identity or changing one's mind? The social context of cognitive dissonance generation and reduction. Swiss Journal of Psychology, 53(4), 202-209.

Cooper, J. M. (2007). Cognitive dissonance: 50 years of a classic theory. London, England: Sage.

Festinger, L. (1957). A theory of cognitive dissonance. Evanston, IL: Row, Petterson.

Fischer, R., Ferreira, M. C., Milfont, T., \& Pilati, R. (2014). Culture of corruption? The effects of priming corruption images in a high corruption context. Journal of Cross-Cultural Psychology, 45(10), 1594-1605. doi: $10.1177 / 0022022114548874$

Fiske, S. T., \& Taylor, S. E. (2013). Social cognition: from brains to culture. London, England: Sage.

Forsyth, D. R., \& Burnette, J. (2010). Group processes. In R. F. Baumeister \& E. J. Finkel (Eds.), Advanced social psychology: the state of the science (pp. 495-534). New York, NY: Oxford University Press.

Gawronski, B., \& Strack, F. (2012). Cognitive consistency as a basic principle of social information processing. In B. Gaweonski \& F. Strack (Eds.), Cognitive consistency: a fundamental principle in social cognition (pp.1-16). New York, NY: Guilford Press.

Glasford, D. E., Dovidio, J. F., \& Pratto, F. (2009). I continue to feel so good about us: in-group identification and the use of social identity-enhancing strategies to reduce intragroup dissonance. Personality \& Social Psychology Bulletin, 35(4), 415-427. doi: 10.1177/0146167208329216

Greenwald, A. G., Banaji, M. R., \& Nosek, B. A. (2003). Understanding and using the Implicit Association Test: I. An improved scoring algorithm. Journal of Personality and Social Psychology, 85(2), 197-216. doi: 10.1037/0022-3514.85.2.197

Greenwald, A. G., \& Farnham, S. D. (2000). Using the Implicit Association Test to measure self-esteem and self-concept. Jounal of Personality and Social Psychology, 79(6), 1022-1038. doi: 10.1037//0022-3514. 79.6.I022

Greenwald, A. G., McGhee, D. E., \& Schwartz, J. L. K. (1998). Measuring individual differences in implicit cognition: the implicit association test. Journal of Personality and Social Psychology, 8, 1464-1480.

Greenwald, A. G., \& Ronis, D. L. (1978). Twenty years of cognitive dissonance: case study of the evolution of a theory. Psychological Review, 85, 53-57.

Greenwald, A. G., Rudman, L. A., Farnham, S. D., Nosek, B. A., \& Mellott, D. S. (2002). A unified theory of implicit attitudes, stereotypes, self-esteem, and self-concept.
Psychological Review, 109(1), 3-25. doi: 10.1037//0033 -295X.109.1.3

Heider, F. (1958). The psychology of interpersonal relations. New York, NY: Wiley.

Jordan, C. H., Spencer, S. J., Zanna, M. P., Hoshino-Browne, E., \& Correll, J. (2003). Secure and defensive high selfesteem. Journal of Personality and Social Psychology, 85(5), 969-978. doi: 10.1037/0022-3514.85.5.969

Jordan, C. H., Logel, C., Spencer, S. J., Zanna, M. P., \& Whitfield, M. L. (2008). The heterogeneity of self-esteem: exploring the interplay between implicit and explicit selfesteem. In R. E. Petty, R. H. Fazio, \& P. Briñol (Eds.), Attitudes: insights from the new implicit measures (pp. 251-284). New York, NY: Psychology Press.

Kernis, M. H., Lakey, C. E., \& Heppner, W. L. (2008). Secure versus fragile high self-esteem as a predictor of verbal defensiveness: converging findings across three different markers. Journal of Personality, 76, 477-512.

Lane, K. A., Banaji, M. R., Nosek, B. A., \& Greenwald, A. G. (2007). Understanding and using the Implicit Association Test. In B. Wittenbrink \& N. Schwarz (Eds.), Implicit measures of attitudes (pp. 59-102). New York, NY: Guilford Press.

Leach, C. W., van Zomeren, M., Zebel, S., Vliek, M. L. W., Pennekamp, S. F., Doosje, B., ... Spears, R. (2008). Grouplevel self-definition and self-investment: a hierarchical (multicomponent) model of in-group identification. Journal of Personality and Social Psychology, 95(1), 144-165. doi: 10.1037/0022-3514.95.1.144

McKimmie, B. M. (2015). Cognitive dissonance in groups. Social and Personality Psychology Compass, 4, 202-212. doi: $10.1111 / \mathrm{spc} 3.12167$

Modesto, J. G. N. (2014). "Nem todas as vítimas importam": a influência das crenças no mundo justo na responsabilização de vítimas de diferentes grupos sociais (Dissertação de Mestrado). Instituto de Psicologia, Universidade de Brasília, Brasília, DF.

Modesto, J., \& Pilati, R. (2015). Implicit deservingness: Implicit Association Test for belief in a just world. Interamerican Journal of Psychology, 49(2), 203-212.

Norton, M. I., Monin, B., Cooper, J., \& Hogg, M. A. (2003). Vicarious dissonance: attitude change from the inconsistency of others. Journal of Personality and Social Psychology, 85(1), 47-62. doi: 10.1037/0022-3514. 85.1 .47

Oliveira, N. R., Janczura, G. A, \& Castilho, G. M. (2013). Normas de alerta e valência para 908 palavras da língua portuguesa. Psicologia: Teoria e Pesquisa, 29, 185-200. doi: 10.1590/S0102-37722013000200008

Petty, R. E., Briñol, P, \& Johnson, I. (2012). Implicit ambivalence. In B. Gawronski \& F. Strack, Cognitive concistensy: a fundamental principle in social cognition (pp. 178-201). New York, NY: Guilford Press.

Robertson, T. (2006). Dissonance effects as conformity to consistency norms: the effect of anonymity and identity salience. British Journal of Social Psychology, 45, 683-699. doi: 10.1348/014466605x82855 
Robertson, T., \& Reicher, S. (1997). Threats to self and the multiple inconsistencies of forced compliance. Social Psychological Review, 1(1), 1-15.

Rosenberg, M. (1965). Society and the adolescent self image. Princeton, NJ: Princeton University Press.

Rudman, L. A., Dohn, M. C., \& Fairchild, K. (2007). Implicit self-esteem compensation: automatic threat defense. Journal of Personality and Social Psychology, 93(5), 798-813. doi: 10.1037/0022-3514.93.5.798

Schnabel, K., \& Asendorpf, J. B. (2010). The self-concept: new insights from implicit measurement procedures. In B. Gawronski \& B. K. Payne, Handbook of implicit social cognition measurement, theory and applications (pp. 392-407). New York, NY: Guilford Press.

Silva, N., Zanelli, J. C., \& Tolfo, S. R. (2013). Diagnóstico e gestão da cultura nas organizações. In L. O. Borges \& L. Mourão, O trabalho e as organizações: atuações a partir da psicologia (pp. 225-252). São Paulo, SP: Artmed.

Smurda, J. D. (2006). Effects of threat to a valued social identity on implicit self-esteem and discrimination.
Group Processes \& Intergroup Relations, 9(2), 181-197. doi: 10.1177/1368430206062076

Stets, J. E., \& Burke, P. J. (2013). Identity theory and social identity theory. Social Psychology Quarterly, 63(3), 224-237.

Tajfel, H. (Ed.). (1978). Differentiation between social groups: studies in the social psychology of intergroup relations. London, England: Academic Press.

Vignoles, V. L., Regalia, C., Manzi, C., Golledge, J., \& Scabini, E. (2006). Beyond self-esteem: influence of multiple motives on identity construction. Journal of Personality and Social Psychology, 90(2), 308-333. doi: 10.1037/0022-3514.90.2.308

Zeigler-Hill, V., \& Jordan, C. H. (2010). Two faces of selfesteem: implicit and explicit forms of self-esteem. In B. Gawronski \& B. K. Payne, Handbook of implicit social cognition measurement, theory and applications (pp. 392-407). New York, NY: Guilford Press.

Recebido: 04/06/2017

Aprovado: 05/02/2018 


\section{Apêndice A}

Itens da Escala de Identificação com o grupo "Brasileiros" (medida em escala Likert de 1 a 7, variando de "discordo completamente" a "concordo completamente")

\begin{tabular}{|c|}
\hline Sou feliz por ser brasileiro. \\
\hline É agradável ser brasileiro. \\
\hline Sinto-me ligado aos brasileiros. \\
\hline Os brasileiros são muito parecidos uns aos outros. \\
\hline Eu acho que os brasileiros têm muito do que se orgulhar. \\
\hline O fato de ser brasileiro é uma parte importante de como eu me vejo. \\
\hline Eu tenho muito em comum com um típico brasileiro. \\
\hline Sinto-me comprometido com os brasileiros. \\
\hline Ser brasileiro me dá uma sensação boa. \\
\hline Eu frequentemente penso sobre o fato de ser brasileiro. \\
\hline Sou parecido com um típico brasileiro. \\
\hline Os brasileiros têm muito em comum uns com os outros. \\
\hline O fato de ser brasileiro é uma parte importante de minha identidade. \\
\hline Sinto-me solidário com os brasileiros. \\
\hline
\end{tabular}

Itens da Escala de Identificação com o grupo "Estudantes universitários" (medida em escala Likert de 1 a 7, variando de "discordo completamente" a "concordo completamente")

\begin{tabular}{|c|}
\hline Sou feliz por ser estudante universitário. \\
\hline É agradável ser estudante universitário. \\
\hline Sinto-me ligado aos estudantes universitários. \\
\hline Os estudantes universitários são muito parecidos uns aos outros. \\
\hline Eu acho que os estudantes universitários têm muito do que se orgulhar. \\
\hline O fato de ser estudante universitário é uma parte importante de como eu me vejo. \\
\hline Eu tenho muito em comum com um típico estudante universitário. \\
\hline Sinto-me comprometido com os estudantes universitários. \\
\hline Ser estudante universitário me dá uma sensação boa. \\
\hline Eu frequentemente penso sobre o fato de ser estudante universitário. \\
\hline Sou parecido com um típico estudante universitário. \\
\hline Os estudantes universitários têm muito em comum uns com os outros. \\
\hline O fato de ser estudante universitário é uma parte importante de minha identidade. \\
\hline Sinto-me solidário com os estudantes universitários. \\
\hline
\end{tabular}




\section{Apêndice B}

\section{Notícia e questões: Brasileiros}

Segundo estudo da Universidade Yale, $80 \%$ dos brasileiros cometem algum tipo de corrupção, liderando o ranking comparativo com outros países.

De acordo com os resultados da pesquisa, encomendada pela ONU, os brasileiros são os que mais se envolvem em comportamentos corruptos no mundo. Foram analisados diversos comportamentos, desde furar fila, não devolver troco, colar em avaliações, pequenos furtos, aproveitamento de posição social para favorecer parentes etc. Especialistas elencam como algumas das principais causas a cultura e as normas sociais vigentes no país. Após a divulgação da pesquisa, em 15/04/2015, aumentou a frequência, em redes sociais, de postagens comparando a crise de corrupção política do país com estes atos corruptos corriqueiros da população.

1. Escreva 5 características (e/ou adjetivos) dos brasileiros que estejam relacionadas ao resultado da pesquisa, indicado na notícia.

2. Nesta atividade você deve cumprir o objetivo a seguir proposto, independente de sua opinião a respeito. Lembre-se de uma situação em que você presenciou a ocorrência de um desses atos corruptos corriqueiros dos brasileiros, ou alguma história que um familiar ou amigo lhe contou, que contenha esses atos, ou que você tenha visto sobre, divulgado em algum lugar. Escreva um paragrafo curto sobre a situação, com o objetivo de alertar turistas sobre os possíveis riscos que eles podem correr ao vir para o Brasil devido à característica predominante nos brasileiros, indicada na notícia, de envolvimento em algum tipo de corrupção. Caso nunca tenha visto, ouvido falar ou lido antes sobre, comente sobre a notícia que acabou de ler para justificar o alerta. 


\section{Apêndice C}

\section{Notícia e questões: Estudantes universitários}

Governo Federal ameaça diminuir repasse de verbas à Universidade de Brasília (UnB) devido a comportamentos impróprios dos universitários.

A Universidade de Brasília conta com o prestígio de ser uma das melhores universidades do país. Contudo, problemas envolvendo o comportamento dos estudantes universitários têm chamado a atenção do Governo Federal. O governo ameaça diminuir o repasse caso alguma intervenção, que vise a adequação dos universitários às normas, não seja efetivada. Os comportamentos dos universitários tidos como impróprios, com base no regulamento da UnB, incluem: mal uso de patrimônio (mesas, cadeiras, quadros, banheiros, etc.); realização de festas não aprovadas pela instituição que atraem não estudantes incorrendo em danos ao patrimônio e riscos aos estudantes; ausência de empenho satisfatório nos estudos o que tem feito as notas de avaliação de diversos cursos diminuir, resultando em queda da UnB no ranking nacional e internacional.

1. Escreva 5 características (e/ou adjetivos) dos estudantes universitários que estejam relacionadas aos problemas indicados na notícia.

2. Nesta atividade você deve cumprir o objetivo a seguir proposto, independente de sua opinião a respeito. Lembre-se de uma situação em que você presenciou, ou um colega lhe contou, ou você viu divulgado em algum lugar, ao menos um dos comportamentos impróprios dos estudantes da UnB comentados na notícia lida. Escreva um parágrafo curto sobre a situação, com o objetivo de alertar estudantes de universidades no exterior, que planejam fazer intercâmbio no Brasil, sobre os possíveis problemas de convivência que podem surgir devido aos problemas de conduta dos estudantes da UnB, além da possibilidade de encontrarem a universidade sucateada devido à diminuição do repasse de verba governamental em decorrência desses problemas de conduta. Caso nunca tenha visto, ouvido falar ou lido antes sobre, comente sobre a notícia que acabou de ler para justificar o alerta. 


\section{Apêndice D}

\section{Notícia: Controle}

Nova espécie de peixe é descoberta por pesquisadores em Mambaí, GO

Uma nova espécie de peixe foi descoberta na Gruta da Tarimba, em Mambaí, a 500km de Goiânia. De acordo com uma das responsáveis pela pesquisa, a professora doutora Maria Elina Bichuette, da Universidade Federal de São Carlos (UFSCar), em São Paulo, o peixe é troglóbio, ou seja, vive exclusivamente em ambientes subterrâneos e só é encontrado naquele local. "A espécie é endêmica e vive apenas nesta gruta. Foram anos de trabalho para que pudéssemos, de fato, comprovar que se trata de um animal raro e que já está ameaçado de extinção", explicou em entrevista ao G1. Segundo a pesquisadora, os primeiros animais foram descobertos em 2004, porém, os estudos só foram concentrados, de fato, entre os anos de 2012 e 2013. Em dezembro do ano passado, o peixe foi oficialmente descrito em um artigo na revista da Sociedade Brasileira de Zoologia.

Fonte: G1. 